\title{
Refitting an X-ray diffraction system for combined GIXRF and XRR measurements
}

\author{
Dieter Ingerle (1), ${ }^{1, a)}$ Werner Artner, ${ }^{2}$ Klaudia Hradil, ${ }^{2}$ and Christina Streli ${ }^{1}$ \\ ${ }^{I}$ TU Wien, Atominstitut, Vienna 1020, Austria \\ ${ }^{2}$ TU Wien, X-Ray Center, Vienna 1060, Austria
}

(Received 25 November 2019; accepted 22 May 2020)

\begin{abstract}
A commercial Empyrean X-ray diffractometer was adapted for combined grazing incidence X-ray fluorescence analysis (GIXRF) measurements with X-ray reflectivity (XRR) measurements. An energy-dispersive silicon drift detector was mounted and integrated in the angle-dependent data acquisition of the Empyrean. Different monochromator/X-ray optics units have been compared with the values obtained by the Atominstitut GIXRF + XRR spectrometer. Data evaluation was performed by JGIXA, a special software for combined GIXRF + XRR data fitting, developed at Atominstitut. A sample consisting of a $\sim 50 \mathrm{~nm}$ nickel layer on a silicon substrate was used to compare the performance criteria (i.e. divergence and intensity) of the incident beam optics. An Empyrean X-ray diffractometer was successfully refitted to measure both GIXRF and XRR data. () The Author(s), 2020. Published by Cambridge University Press on behalf of International Centre for Diffraction Data. This is an Open Access article, distributed under the terms of the Creative Commons Attribution licence (http://creativecommons.org/licenses/by/4.0/), which permits unrestricted re-use, distribution, and reproduction in any medium, provided the original work is properly cited.
\end{abstract}

[doi:10.1017/S088571562000041X]

Key words: GIXRF, XRR, thin-film characterization, X-ray optics

\section{INTRODUCTION}

$\mathrm{X}$-ray reflectometry (XRR) is a well-known and established technique for the characterization of single- and multi-layered thin-film structures with layer thicknesses in the nanometer range. XRR spectra are acquired by varying the incident angle in the grazing incidence regime while measuring the intensity of the specular reflected X-ray beam. The shape of the resulting angle-dependent curve is correlated to changes of the electron density in the sample and, specifically in the case of layers, distinct Kiessig fringes can be observed (Kiessig, 1931). The position and intensity of these fringes can be calculated (Parratt, 1954) and thus be used for the characterization of layered samples.

Grazing incidence X-ray fluorescence (GIXRF) is a total reflection X-ray fluorescence analysis (TXRF) related technique, which uses the angle-dependent XRF signal in the grazing incidence regime. While TXRF uses measurements at a single point below the critical angle, GIXRF uses angle scans from $0^{\circ}$ up to 3 or 4 times the critical angle in order to evaluate the angle-dependent variations in the X-ray standing wavefield. The XRF signal is element-specific, and therefore, the measurements contain information about the elemental composition, concentration profile, and thickness and density of near-surface layers (de Boer, 1991).

The combined measurement and evaluation of GIXRF and XRR data can improve the obtained information, as it reduces uncertainties and ambiguities of the individual techniques, especially for the analysis of samples, for which the exact stoichiometry might not be known (Ingerle et al., 2014a).

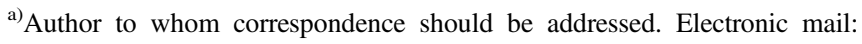
dieter.ingerle@tuwien.ac.at
}

Already in 1994, van den Hoogenhof and de Boer, while working at Philips, presented a setup for combined GIXRF and XRR experiments (van den Hoogenhof and de Boer, 1994). Nevertheless and although there are several manufacturers for TXRF (or limited GIXRF) instrumentation or for diffractometers, which can be used for XRR analysis, at the moment there is no commercial instrument available, which (out-of-the-box) provides the possibility to perform combined GIXRF/XRR analysis.

In a previously published approach, we modified an existing GIXRF setup by adding a detector for the simultaneous acquisition of XRR intensities (Ingerle et al., 2014b). Although this setup has several advantages (e.g. vacuum chamber and easily exchangeable tube anode material), it also suffers from a somewhat limited measurement resolution because of minimum motor step size as well as beam divergence. As an improvement in this respect would amount to a complete redesign, we considered a different approach and looked at the instruments, which are available at the X-ray Center of our university.

We used an Empyrean X-ray diffraction (XRD) system by PANalytical, which offers beam optics, detectors, and software for XRR on thin layers, but no support for the acquisition of X-ray fluorescence, and added an Amptek silicon drift detector (SDD) for the acquisition of XRF spectra (Figure 1). A custom control software was developed in order to synchronize the acquisition of XRF spectra with the angular movement during an XRR scan.

\section{EXPERIMENTAL}

The Empyrean XRD system by PANalytical is a commercially available platform for a variety of applications in analytical XRD. The goniometer, which is the central part of the 


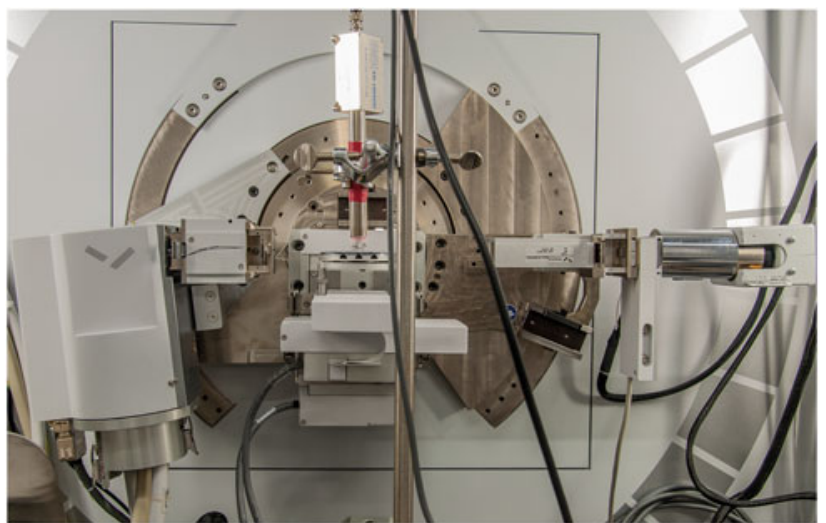

Figure 1. (Color online) Empyrean setup with the XRF detector (Amptek SDD).

diffractometer, has a radius of $240 \mathrm{~mm}$. It features Heidenhain encoders and a minimum step size of $0.0001^{\circ}$ for the angle of incidence as well as the scattering angle. The instrument uses a $\theta / \theta$ configuration, i.e. the sample is stationary in the horizontal position, whereas the tube and XRR detector move simultaneously. The PreFIX concept allows the exchange of beam optical and detector modules within minutes.

Specifics about the used components were taken from the Empyrean Reference Manual.

\section{A. X-ray tube}

The line focus of an Empyrean Cu LFF HR X-ray tube was used for the measurements. This metal-ceramic tube has a maximum power rating of $1.8 \mathrm{~kW}$ and a focal spot of $12 \times 0.4 \mathrm{~mm}$. The exit window consists of beryllium with a thickness of 300 $\mu \mathrm{m}$. The tube was operated at $45 \mathrm{kV}$ and $40 \mathrm{~mA}$.

\section{B. X-ray optics}

A wide variety of incident beam optics, i.e. monochromators and mirrors, is available for the Empyrean. We used and compared four of them:

- The hybrid monochromator consists of a parabolical shaped graded multilayer and a channel-cut $\mathrm{Ge}(220)$ crystal in one module. It creates almost pure $K \alpha_{1}$ radiation. The $K \alpha_{2}$ radiation is reduced to below $0.1 \%$ of the original value.

- The parallel beam X-ray mirror module contains a parabolical shaped graded multilayer. It converts the divergent beam into a monochromatic $K \alpha$ quasi-parallel beam. The $K \beta$ radiation is reduced to below $0.5 \%$ of the original value.

- The Bragg-BrentanoHD module converts a divergent X-ray beam into a monochromatic divergent X-ray beam. We used this module with a $1 / 32^{\circ}(0.05 \mathrm{~mm})$ exit slit. The energy resolution is about $450 \mathrm{eV}$.

- The focusing X-ray mirror module contains an ellipticalshaped graded multilayer. It converts the divergent beam into a monochromatic $K \alpha$ beam, focused on the detector. The $K \beta$ radiation is reduced to below $0.5 \%$ of the original value.

All modules were used with a $1 / 32^{\circ}(0.05 \mathrm{~mm})$ divergence slit and a $10 \mathrm{~mm}$ wide beam mask.

\section{XRR detector}

The XRR detector assembly consists of a $0.18^{\circ}$ parallel plate collimator, a $0.1 \mathrm{~mm}$ collimator slit, a programmable beam attenuator, and the detector module. The beam attenuator contains a Nickel foil, which is $125 \mu \mathrm{m}$ thick. The foil, which reduces the $\mathrm{Cu} K \alpha$ intensity by a factor of 174 , is automatically inserted into or retracted from the beam path if the count rate exceeds or falls below a configured threshold.

Concerning the detector module, we tested a scintillation and a PIXcel ${ }^{3 \mathrm{D}}$ detector, which is based on Medipix 2 technology (Llopart et al., 2002). After measurements with the parallel beam mirror, we realized that the count rate at small angles exceeds the specified $99 \%$ linearity range of the scintillation detector (0-500 kcps). Thus, we only used the PIXcel3D for our comparison, which has a $99 \%$ linearity range of $0-5 \times 10^{6} \mathrm{cps}$ per column. The detector consists of $255 \times 255$ px with a pixel pitch of 55 $\mu \mathrm{m}$ and was used in the open detector (0D) mode.

\section{XRF detector}

An Amptek XR-100SDD with an $8 \mu \mathrm{m}$ thick Beryllium entrance window was placed at $90^{\circ}$ to the sample surface (Figure 1). The detector has a $25 \mathrm{~mm}^{2}$ active area (internally collimated to $17 \mathrm{~mm}^{2}$ ) and is $500 \mu \mathrm{m}$ thick. The signal processing was done by an Amptek PX4 digital pulse processor (DPP). This combination of SDD and DPP results in an approximate maximum input count rate of $200 \mathrm{kcps}$ at 2.4 $\mu$ s peaking time and a minimal achievable resolution of 125 $\mathrm{eV}$ at $5.9 \mathrm{keV}$ (Amptek, 2020). As GIXRF measurements can imply high count rates above the critical angle and XRF peak overlaps in case of multi-element samples, good count rate capability as well as energy resolution are both critical.

During the mounting, care has to be taken that the center of the XRF detector aligns with the center of rotation in order to make the measurements as accurate and reproducible as possible. The location and stability of the center of rotation of the Empyrean system can be verified by a fluorescence disk, which is provided with the diffractometer. Furthermore, we used distance holders to place the detector $3 \mathrm{~mm}$ above the sample surface.

The total efficiency, which in the considered energy range up to $8 \mathrm{keV}$ is mainly influenced by the absorption in the air path and the Beryllium window, can be estimated to $\sim 50 \%$ for $\mathrm{SiK} \alpha$ at $1.74 \mathrm{keV}$ and $>95 \%$ above $4 \mathrm{keV}$.

\section{E. Acquisition software}

We used the Data Collector program, which is the standard software for the Empyrean, for movement control and for the acquisition of XRR data. In order to start, stop, and read out the XRF detector in synchrony with the XRR scan, we developed our own software.

This was actually the most challenging task in the adaption of the diffractometer, as the commercial control software acts more or less like a black box, with no documentation on programming interfaces available to us. Fortunately, the communication between the diffractometer and control software is unencrypted via plain text serial port connection. Thus, we were able to write a software module, which intercepts this communication and forwards information on angle positions and scan status to our own control software, which manages the XRF detector acquisition. One limitation of this approach is a slight overhead of 1-2 s, which we had to introduce at each angle step, in order to facilitate the timely stopping of the XRF detector. Furthermore, the XRF scan acquisition is 
only started for symmetric goniometer scans in the step mode, as continuous scans would introduce additional inaccuracies.

\section{RESULTS AND DISCUSSION}

\section{A. Comparison of beam optics}

A sample consisting of a $\sim 50 \mathrm{~nm}$ nickel layer on a silicon substrate was used to compare the performance (i.e. divergence and intensity) of the incident beam optics. We performed combined measurements of $\mathrm{SiK} \alpha \mathrm{XRF}$ and XRR with an acquisition time of $7 \mathrm{~s}$ per point. The NiKo XRF from the layer is not accessible with the copper tube and the available optics as the Ni-K edge $(8.3 \mathrm{keV})$ is above the energy of the $\mathrm{Cu} K \alpha$ radiation $(8.04 \mathrm{keV})$, but it is important to note that the $\mathrm{SiK} \alpha$ GIXRF from the substrate is also modulated because of the interference effects caused by the layer. The evaluation was done with the software JGIXA (Ingerle et al., 2016).

We used two figures of merit to evaluate and compare the performance of the different optics: The measured intensities and the divergence reported by the evaluation software.

The intensities (i.e. count rates), which are measured by the XRF and XRR detector, are directly correlated to the intensity of the beam after the primary optics. Typically, one would aim for the highest possible intensity in order to reduce counting times especially for larger angles in the XRR spectrum or for the XRF measurement of low concentration samples. Nevertheless, we have to add the following caveat: the intensity can be too high for the chosen detector and sample combination. For example, as mentioned before, the intensities of the parallel and focusing beam mirrors were actually exceeding the specified linearity range of the scintillation detector of the Empyrean system even when an attenuator foil was inserted. This effect leads to a damping of the total reflection region in the spectrum and, thus, a distortion of the critical angle or an underestimation of Bragg-peaks. A similar effect can be observed for the XRF measurement of elements with high concentration and good cross-section for the incident radiation. In this case, mainly, the angles above the critical angle, i.e. when the beam is fully penetrating into the sample, are affected. The evaluation of all elements in the XRF spectrum will be impaired, leading to wrong quantification results. In case no better detector is available, these adverse conditions can be avoided by reducing the tube current or, maybe even better, by choosing a different X-ray optics, which has reduced flux, but might create a more coherent beam, as was observed in our experiments (see below).

The reported divergence has to be considered as a measure for the coherence of the beam relevant for the experiment and not as a full characterization of the beam. XRR and GIXRF are based on beam interference effects and, thus, rely on a suitable coherence to show oscillations in the angle-dependent measurement curve. We can distinguish two types of coherence, which might be relevant for the techniques: Firstly, we have the temporal coherence and, secondly, the spatial coherence, which can be described by the monochromaticity and the angular divergence of the beam, respectively. The angular divergence can be subdivided in two values for the planes parallel and perpendicular to the sample surface, but in the case of XRR and GIXRF, it is mainly the angle distribution perpendicular to the surface, which is of relevance for the measurement.
Further discussion on this topic can be found in the literature (von Bohlen et al., 2009; Tiwari et al., 2015).

In our setup, we have an X-ray tube as the primary source, which has to be considered as an incoherent source. We use monochromators, i.e. crystals or multilayers, to improve the temporal coherence and slits or collimators to improve the spatial coherence. Special cases are multilayers on a bent or shaped substrate, graded multilayers, or bent or shaped crystals, as they will improve temporal and spatial coherence at the same time, i.e. typically a parallel or focused, monochromatic beam. One fact, which is working to the advantage of $\mathrm{X}$-ray tubes used with multilayers, is the use of characteristic lines of the anode material. Although multilayers typically have a bandwidth of some hundred $\mathrm{eV}$ near $\mathrm{CuK \alpha}(\sim 8$ $\mathrm{keV}$ ), the actual monochromatization is still sufficient for many experiments, as the main component of the beam will be $K \alpha_{1}$ and $K \alpha_{2}$ radiation. As these two lines are only $\sim 20$ $\mathrm{eV}$ apart, the additional error, introduced by the shift in angles at different energies, is typically negligible for many samples in comparison to the much larger angular divergence.

Considering this, we assume perfectly monochromatic excitation for the simulation in JGIXA and introduce a convolution with a Gaussian point spread function in order to simulate the angular spread of the beam. Furthermore, the coherence for the XRR measurement can be further improved by additional optical elements after the sample and in front of the detector. Thus, the simulation uses two separate values for the simulation of divergence in the GIXRF and the XRR calculations.

Coming back to our comparison of the optics, we clearly see an expected tradeoff between intensity and estimated divergence (Figure 2). The divergence for the XRR measurement seems to be reduced because of the additional slit and a parallel plate collimator in front of the XRR detector. A closer look at some details in the measurement curves clearly shows the benefit of the better divergence (Figure 3). The comparison with a self-built spectrometer for combined GIXRF and XRR measurements (Ingerle et al., 2014b), which only uses a flat multilayer and a slit system, is also instructive, as it is clear to see, that features start to disappear, which are visible with the hybrid monochromator of the Empyrean.

\section{B. Importance of divergence for GIXRF measurements}

In the past, we successfully used a modified GIXRF setup for combined GIXRF and XRR measurements (Ingerle et al., 2014b). The analyzed samples mainly involved the depthprofiling of shallow depth ion-implantation (Ingerle et al., 2014a) or diffusion effects because of annealing in thin layers (Caby et al., 2015; Rotella et al., 2017). All of these samples could not be analyzed by XRR alone, as the change in the electron density was almost non-existent, either due the low implantation dose or the similarity in the atomic number of the involved elements. Furthermore, the divergence was not a problem for these studies, as the critical features of the measurement curves were not expected to be significantly influenced.

But in a recent analysis, the divergence was expected to become a problem for the evaluation of the measurement. This gave us the opportunity to test the new approach for a combined setup, which is presented in this manuscript.

The analyzed samples consisted of a simplified model built from typical materials for organic light-emitting diode 

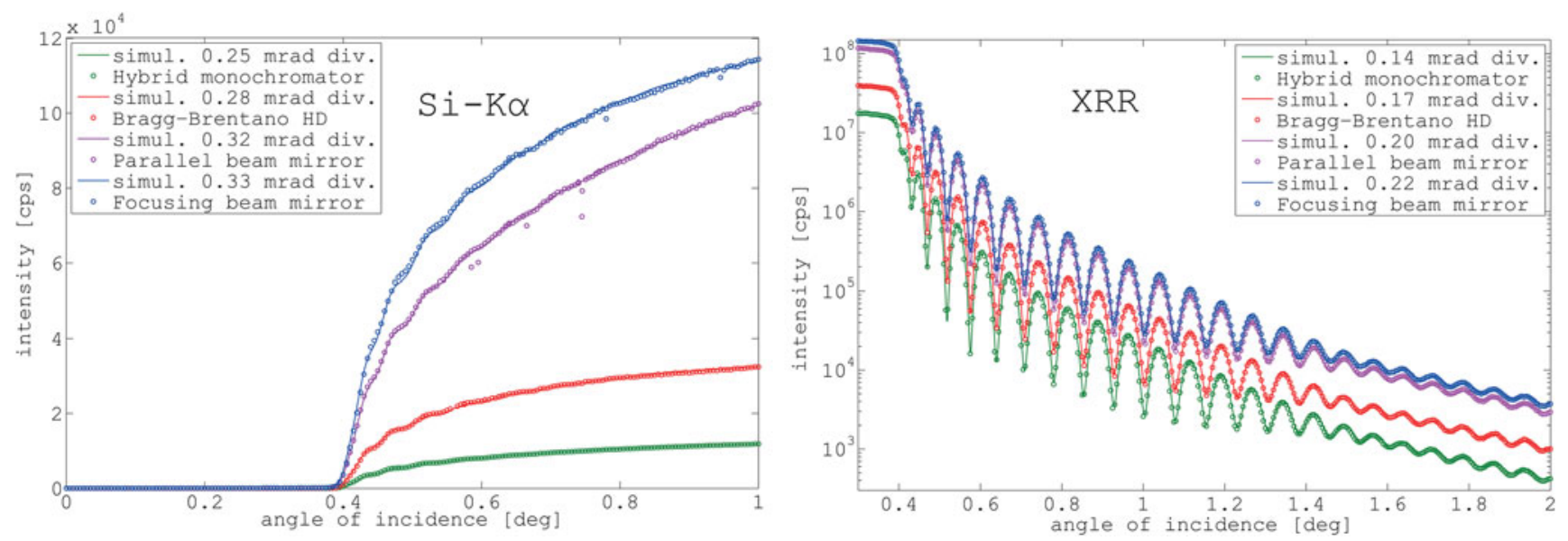

Figure 2. (Color online) Intensity of $\mathrm{SiK \alpha}$ signal (left) and X-ray reflectivity intensity (right) versus angle of incidence for the various X-ray optics of the Empyrean diffractometer.

(OLED) production, i.e. mainly organic layers with a sulfur based small molecule host. The topic of interest was the difference in diffusion because of vapor or solution-based deposition. Here again, the analysis with XRR alone would provide inconclusive results, as the concentration of sulfur is small $(\sim 1-2 \%)$ and also the variation in the distribution is very small and thus results in almost no change of electron density.

Further information on the result and the samples can be found in another publication (Maderitsch et al., 2018).

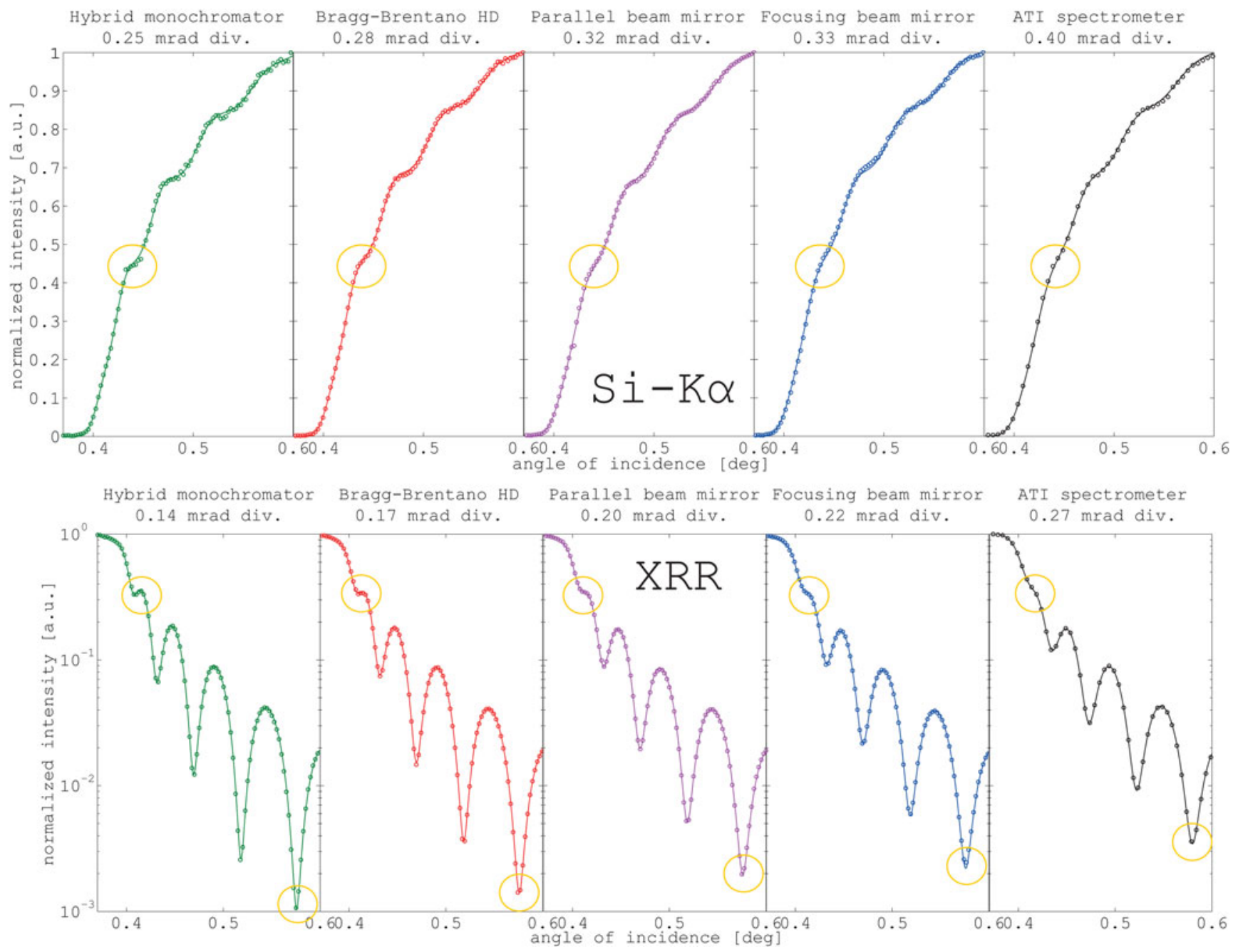

Figure 3. (Color online) Intensity of SiKo signal (top) and X-ray reflectivity intensity (bottom) versus angle of incidence for the various X-ray optics of the Empyrean diffractometer. The angle range from 0.4 to $0.6^{\circ}$ is plotted to emphasize the influence of the divergence of the used X-ray optics on specific features (marked with a circle). Additionally, the results from the ATI GIXRF + XRR spectrometer are given. The divergence values are the results of the fitting software JGIXA. 

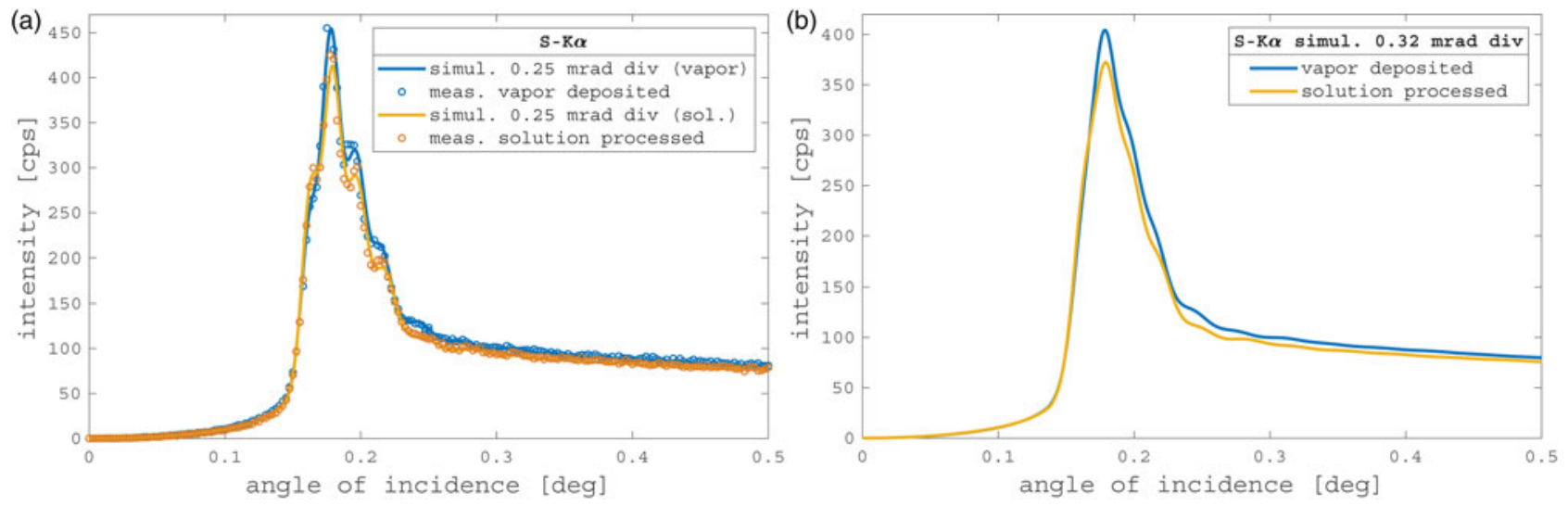

Figure 4. (Color online) Intensity of SK $\alpha$ of an OLED model system with differently prepared host layer. (a) The actual measurement with the hybrid monochromator and a simulation with $0.25 \mathrm{mrad}$ divergence; (b) simulations of the same sample models as in (a), but with $0.32 \mathrm{mrad}$ divergence. The distinct steps in the curves, which are clearly visible in (a), are only slightly noticeable because of the increased divergence.

We want to showcase here the measurement of an OLED model system consisting of a $20 \mathrm{~nm}$ buffer layer, a $20 \mathrm{~nm}$ hole transport layer, and a $60 \mathrm{~nm}$ host layer, with a special emphasize on the influence of the divergence on the measurement curve. The variation in the angle-dependent $\mathrm{S} K \alpha$ intensities can be used to conclude on the distribution of sulfur in the differently prepared host layers (solution processed or evaporated), but only if we can discern the features in the measured curves.

Figure 4(a) shows the actual measurements of the samples with the hybrid monochromator, which were fitted with a simulated divergence of $0.25 \mathrm{mrad}$. Several features, i.e. steps in the rising and falling edge, are clearly visible in the measurement and well-matched in the simulation. If we perform a simulation with $0.32 \mathrm{mrad}$ divergence, which corresponds to the divergence expected from a parallel beam mirror, we can see that the features become much harder to notice [Figure 4 (b)]. In fact, the modulations in intensity are so small, that they could be mistaken for effects of counting statistics. From this comparison, it is clear that a measurement with the parallel beam mirror would make it much harder if not impossible to perform an evaluation and the hybrid monochromator is much better suited for this analysis.

\section{CONCLUSION}

- Our work demonstrates that it is relatively easy to integrate an XRF detector for GIXRF measurements into a commercially available XRD/XRR setup. This fact in combination with an evaluation software creates the opportunity for new applications and users.

- The beam optics worked as expected, showing a tradeoff between divergence and intensity. In the current configuration, the Bragg-BrentanoHD module seems to represent a good compromise for most applications.

- The setup was successfully used for the measurement of organic multilayer structures (Maderitsch et al., 2018). This analysis required the better resolution of the hybrid monochromator.

Amptek (2020). XR-100SDD Silicon Drift Detector (SDD). Available at: https://www.amptek.com/products/sdd-x-ray-detectors-for-xrf-eds/ xr-100sdd-silicon-drift-detector (Retrieved March 25, 2020).
Caby, B., Brigidi, F., Ingerle, D., Nolot, E., Pepponi, G., Streli, C., Lutterotti, L., André, A., Rodriguez, G., Gergaud, P., Morales, M., and Chateigner, D. (2015). "Study of annealing-induced interdiffusion in $\operatorname{In}_{2} \mathrm{O}_{3} / \mathrm{Ag} / \mathrm{In}_{2} \mathrm{O}_{3}$ structures by a combined $\mathrm{X}$-ray reflectivity and grazing incidence $\mathrm{X}$-ray fluorescence analysis," Spectrochim. Acta, Part B 113, 132-137.

de Boer, D. K. G. (1991). "Glancing-incidence X-ray fluorescence of layered materials," Phys. Rev. B 44(2), 498-511.

Ingerle, D., Meirer, F., Pepponi, G., Demenev, E., Giubertoni, D., Wobrauschek, P., and Streli, C. (2014a). "Combined evaluation of grazing incidence X-ray fluorescence and X-ray reflectivity data for improved profiling of ultra-shallow depth distributions," Spectrochim. Acta, Part B 99 (100), 121-128.

Ingerle, D., Schiebl, M., Streli, C., and Wobrauschek, P. (2014b). "Combination of grazing incidence X-ray fluorescence with X-ray reflectivity in one table-top spectrometer for improved characterization of thin layer and implants on/in silicon wafers," Rev. Sci. Instrum. 85(8), 083110.

Ingerle, D., Pepponi, G., Meirer, F., Wobrauschek, P., and Streli, C. (2016). "JGIXA - A software package for the calculation and fitting of grazing incidence X-ray fluorescence and X-ray reflectivity data for the characterization of nanometer-layers and ultra-shallow-implants," Spectrochim. Acta, Part B 118, 20-28.

Kiessig, H. (1931). "Interferenz von Röntgenstrahlen an dünnen Schichten," Ann. Phys. 402(7), 769-788.

Llopart, X., Campbell, M., Dinapoli, R., San Segundo, D., and Pernigotti, E. (2002). "Medipix2: A 64-k pixel readout chip with 55- $\mu \mathrm{m}$ square elements working in single photon counting mode," IEEE Trans. Nucl. Sci. 49(5), 2279-2283.

Maderitsch, A., Ingerle, D., Bretschneider, T., Rauwolf, M., Pflumm, C., Buchholz, H., Borchert, H., Streli, C., and Parisi, J. (2018). "Analysis of organic multilayer structures using a combined grazing incidence X-ray fluorescence/X-ray reflectometry approach," Spectrochim. Acta, Part B 148, 188-192.

Parratt, L. (1954). "Surface studies of solids by total reflection of X-rays," Phys. Rev. 95(2), 359-369.

Rotella, H., Caby, B., Ménesguen, Y., Mazel, Y., Valla, A., Ingerle, D., Detlefs, B., Lépy, M. C., Novikova, A., Rodriguez, G., Streli, C., and Nolot, E. (2017). "Elemental depth profiling in transparent conducting oxide thin film by X-ray reflectivity and grazing incidence X-ray fluorescence combined analysis," Spectrochim. Acta, Part B 135, 22-28.

Tiwari, M. K., Das, G., and Bedzyk, M. (2015). "X-ray standing wave analysis of nanostructures using partially coherent radiation," Appl. Phys. Lett. 107 (10), 103104.

van den Hoogenhof, W. W., and de Boer, D. K. G. (1994). "Glancing incidence X-ray analysis: Forgotten or to be discovered?" Surf. Interface Anal. 22(1-12), 572-575.

von Bohlen, A., Krämer, M., Sternemann, C., and Paulus, M. (2009). "The influence of X-ray coherence length on TXRF and XSW and the characterization of nanoparticles observed under grazing incidence of X-rays," J. Anal. At. Spectrom. 24(6), 792. 